\title{
DETERMINATIONS OF SOME TEXAS COLEOPTERA, WITH RECORDS.
}

By Elbert S. Tucker, Museum Assistant in Systematic Entomology, University of Kansas, Lawrence.

$\mathrm{O}^{\mathrm{N}}$ account of personal interest in the insect fauna of Texas, due mainly to my first entomological collecting trip in the state ( to Galveston, in May, 1904, as a member of a party under direction of Dr. F. H. Snow, for the University of Kansas), the writer consented to identify some beetles from the collection of the Agricultural and Mechanical College of Texas, at College Station, Brazos county, on the request of Prof. A. F. Conradi, then acting state entomologist. As a motive for the work other than the advantage accruing to the college collection, Professor Conradi proposed that a list of determinations thus obtained would be helpful in making up a general list of the Coleoptera of Texas, and the names and records herewith show the results of study of the specimens which he furnished. All determinations were personally made by comparison with named specimens in the collection of beetles of the University of Kansas, together with the aid of literature, excepting those species so indicated in the list, where special credit is given to the authority to whom the specimens were submitted in cases of uncertainty. With the exception of Balaninus victoriensis Chitt., all of the beetles were collected in Texas, mostly at the Agricultural and Mechanical College, to which institution they have been returned, less a few duplicates retained by kind permission. The student will notice that many dates of collecting occurred in early spring and, in fact, winter months, which indicates that where mild winters prevail a long season for insect activity is permitted.

The arrangement follows Henshaw's check-list by numbers when given.

\section{Family CicindeLIDE.}

$19 a$ Cicindela scutellaris Say, var. unicolor Dejean. Wellborn, Brazos river, March 22, 1904.

32 Cicindela vulgaris Say. Wellborn, Brazos river, March 22, 1904.

Family CARABID $\approx$.

184 Calosoma lugubre Le Conte. College Station.

220 Scarites subterraneus Fabricius. College Station, March 31, 1904.

387 Bembidium variegatum Say. College Station, May 9, 1903. 
617 Evarthrus heros Say. Comanche, March 17, 1904.

$847 a$ Casnonia pennsylvanica Linnæus, var. suturalis Chaudoir. College Station, January 30, 1903; Buffalo Springs, May 5, 1903.

935 Pinacodera platicollis Say. College Station, January 4, 1904. Striæ not deep; abdomen protrudes farther beyond elytra than with compared examples, regarding which Mr. Charles Liebeck has written: "About the usual form; the extent of abdominal segment beyond the elytra is variable."

1068 Discoderus impotens Le Conte. College Station, May 1, 1904. Determined by Charles Liebeck.

1120 Harpalus gravis Le Conte. Waco, September 20, 1902. Collected by L. H. Scholl.

1201 Anisodactylus piceus Le Conte. College Station, February 27 and May 1, 1904; Avalon, April 12, 1904. The brownish-tinged examples were probably freshly emerged when captured; hence, had not developed to black.

\section{Family GYRINIDA.}

1539 Dineutes carolinus Le Conte. College Station.

Family CoccinelLid $\approx$.

3064 Cycloneda sanguinea Linnæus. College Station, May 12, 1904 ; also May 10, 1905 (C. E. Sanborn). Predaceous on plant-lice.

$3065 a$ Olla abdominalis Say. Sabinal, May 24, 1904.

3084 Exochomus constritatus Mulsant. College Station, May 10, 1905 (C. E. Sanborn). Predaceous on plant-lice.

3098 Brachyacantha bollii Crotch. College Station, June 4, 1903. Determined by Charles Liebeck as "large form." The yellow spaces of basal and median portions of elytra are solidly fused, without any indication of spots. Also taken at College Station, May 10, 1905, by C. E. Sanborn, who reported it as predaceous on plant-lice.

3135 Scymnus terminatus Say. College Station, August 8, 1904.

3156 Scymnus tenebrosus Mulsant. College Station, May 10, 1905 (C. E. Sanborn). Predaceous on plant-lice.

Family ERotylid A.

3202 Languria tædata Le Conte. College Station, April 12, 1903.

Family Elaterid $A$.

4085 Lacon rectangularis Say. College Station, February 27, 1904.

Family BUPRESTIDÆ.

4575 Psiloptera drummondi Laporte and Gory. College Station, June 20, 1902. Determined by Charles Liebeck.

4639 Chrysobothris femorata Fabricius, var. alabamæ Gory. Kosse, April 19, 1904.

4750 Agrilus muticus Le Conte. College Station, April 28, 1903, and April 7, 1904.

Family MaLACHIDA.

5007 Collops balteatus Le Conte. Wellborn, Brazos river, March 29, 1903; College Station, July 27, 1902.

\section{Family CleRID E.}

$5215 a$ Chariessa pilosa Say, var. onusta Say. College Station, April 13, 1903. 
Family PTINIDж.

5356 Amphicerus bicaudatus Say. Corsicana, March 11, 1904.

Family SCARABæID

5535 Aphodius serval Say. College Station, January 7, 1904. Smaller than compared examples, more deeply striate with confluent punctures, and with slightly different markings, regarding which Mr. Charles Liebeck has written: "About the usual size ; markings variable." 5581 Ochodæus biarmatus Le Conte. Sabine, May 24, 1904.

5591 Bolboceras lazarus Fabricius. College Station, April 14, 1904.

5691 Macrodactylus angustatus Beauvois. College Station, March 30, 1904. 5692 Macrodactylus uniformis Horn. College Station, March 30, 1904. 5742 Lachnosterna torta Le Conte. College Station, July 8, 1904. 5894 Allorhina nitida Linnæus. College Station, July 23, 1904. 5901 Euphoria kernii Haldeman, (typical). Cedars, May 28, 1904. 5941 Trichius viridulus Fabricius. Wellborn, Brazos river, March 30, 1904.

Family Cerambycide.

5953 Mallodon dasystomus Say. College Station.

5955 Mallodon serrulatus Le Conte. Wellborn, Brazos river, June 24, 1904. 5962 Prionus inbricornis Linnæus. College Station, May 1, 1904.

6008 Callidium antennatum Newman. College Station, February 5, 1903.

6038 Chion cinctus Drury. Victoria, August 13, 1904.

6050 Romaleum atomarium Drury. College Station, June 10, 1904.

6115 Callichroma plicatum Le Conte. No data.

$6127 a$ Tragidion coquus Linnæus, var. fulvipenne Say. College Station, February 16, 1903.

6196 Neoclytus luscus Fabricius. Wellborn, Brazos river, June 24, 1904.

6201 Neoclytus erythrocephalus Fabricius. Wellborn, Brazos river, August 12, 1904.

6471 Ataxia crypta Say. Manor, October 5, 1904.

Family Chrysomelide.

6725 Fidia viticida Walsh. No data.

10352 Fidia cana Horn. Dripping Springs, May 17, 1904. Determined by Charles Liebeck. Badly crushed and broken.

6800 Chrysomela disrupta Rogers. Courtney, October 7, 1902. Collection of L. H. Scholl.

6821 Chrysomela auripennis Say. College Station, December 12, 1903.

6978 Haltica nana Crotch. College Station, September 19, 1903. Verified by Charles Liebeck.

7032 Mantura floridana Crotch. College Station, May 1, 1903.

7051 Chætocnema opacula Le Conte. College Station, May 1, 1903.

7055 Chætocnema confinis Crotch. College Station, May 1, 1903.

Family Tenebrionid

7179 Conœeus ovipennis Horn. Comanche, August 17, 1901. Determined by Charles Liebeck.

Emmenastus convexus Le Conte. Coleman, August 23, 1902.

7201 Epitragus acutus Le Conte: Sabinal, May 24, 1904.

7359 Eleodes seriata Le Conte. College Station.

7426 Opatrinus aciculatus Le Conte. College Station, December 20, 1903. 
7438 Blapstinus pratensis Le Conte. Cororado, August 11, 1903 ; College Station, April 12, 1903, and February 27, 1904.

7573 Helops farctus Le Conte. College Station, June 10, 1904.

\section{Family MoRDELLID E.}

7780 Mordella scutellaris Fabricius. College Station, September 16, 1902.

Family ANTHICID $\approx$.

7869 Eurygenius wildii Le Conte. No data.

Family MeLOID E.

8018 Henous confertus Say. College Station, May 28, 1903 ; Wellborn, Brazos river, June 24, 1904.

8083 Epicauta sericans Le Conte. Montgomery, August 24, 1903.

8123 Pomphopœa texana Le Conte. Thornberry, April 4, 1903.

Family Attelabid $\nRightarrow$.

8224 Attelabus analis Illiger. College Station, July 27, 1904.

Family OTIORHYNCHIDÆ.

8242 Ophryastes vittatus Say. Comanche, November 14, 1904.

8326 Achrastenus griseus Horn. Lovelady, March 24, 1904; Grape Vine, no data; Sidney, April 4, 1904. Determined by Charles Liebeck.

8340 Eudiagogus pulcher Fahræus. Wellborn, Brazos river, August 27, 1904 ; College Station, January 30, 1903.

\section{Family CURCULIONIDÆ.}

8370 Apion ovale Smith. Rockdale, June 22, 1901.

8478 Pachylobius picivorus Germar. Hughes Springs, May 11, 1904 ; College Station, June 10, 1904 ; De Kalb, August 1, 1904. The last was in prime condition, neither rubbed nor smeared.

8546 Desmoris constrictus Say. College Station; Center Point, June 1, 1904. Regarding this species Prof. A. F. Conradi has written: "This species occurs abundantly over the entire settled portions of the state of Texas definitely known.",

8615 Magdalis barbiti Say. Howe, March 22, 1904.

10985 Anthonomus grandis Boheman. College Station, June 12, 1904.

8726 Conotrachelus naso Le Conte. College Station, October 20, 1904.

8741 Rhyssematus palmacollis Say. Frost, May 28, 1904. "Hillsboro, Malakoff, Victoria, and in fact it occurs all over the state," writes Professor Conradi. Variety, with costæ, apical margin and spots on elytra rusty red colored. Verified by Charles Liebeck.

8746 Chalcodermus æneus Boheman. Denison, June 27, 1904.

8760 Acalles porosus Le Conte. San Antonio, March 30, 1904 ; Martindale, April 14, 1902.

8902 Aulobaris ibis Le Conte. College Station, December 18, 1904.

- Balaninus victoriensis Chittenden. Mead, Indian Territory, September 20, 1904; Lone Wolf, Oklahoma, September, 1904. Determined by F. H. Chittenden.

Family BRENTHID

8967 Eupsalis minuta Drury. Wellborn, Brazos river, June 24, 1904. 
Family CALANDRID $F$.

8990 Sphenophorus cariosus Olivier. Moody, March 25, 1904. Determined by F. H. Chittenden.

9006 Sphenophorus compressirostris Say. No data. Determined by F. H. Chittenden.

Family ANTHRIBIDÆ.

9222 Cratoparis lugubris Olivier. College Station, January 4, 1904.

SUmmary : Species personally determined $\ldots \ldots \ldots \ldots .76$

Species specially determined........... 9

Number of species reported.......... $\frac{\overline{85}}{85}$ 


\section{$2 \mathrm{BHL}$ Biodiversity Heritage Library}

Tucker, Elbert Stephen. 1906. "Determinations of some Texas Coleoptera with records." Transactions of the Kansas Academy of Science 20, 85-89. https://doi.org/10.2307/3624688.

View This Item Online: https://www.biodiversitylibrary.org/item/35329

DOI: https://doi.org/10.2307/3624688

Permalink: https://www.biodiversitylibrary.org/partpdf/2887

\section{Holding Institution}

Harvard University, Museum of Comparative Zoology, Ernst Mayr Library

\section{Sponsored by}

Harvard University, Museum of Comparative Zoology, Ernst Mayr Library

\section{Copyright \& Reuse}

Copyright Status: NOT_IN_COPYRIGHT

This document was created from content at the Biodiversity Heritage Library, the world's largest open access digital library for biodiversity literature and archives. Visit BHL at https://www.biodiversitylibrary.org. 